\title{
Research on mobile robot navigation based on Kinect depth information
}

$$
\text { Lizong Lin }{ }^{1, a} \text {, Hao Zhang }{ }^{2, b} \text {, Yajun } \mathrm{Xu}_{\mathrm{Liu}^{3, \mathrm{e}}}^{3, \mathrm{c}} \text {, Changxian Zhang }{ }^{4, \mathrm{~d}} \text { and Shuang }
$$

Department of Mechanical Engineering, East China University of Science and Technology, Shanghai 200237, China

azzlin@ecust.edu.cn, ${ }^{\text {b}} 429279495 @ q q . c o m$

Keywords: Kinect, Mobile robot, SLAM, Path planning

Abstract. This paper presents a method of mobile robot navigation based on the Kinect. The proposed method has advantages of flexibility and low cost compared with the traditional sensors, such as laser scanner. Using the Kinect depth information, the SLAM and path planning are realized and the mobile robot navigation is studied. Simulation is performed on two-wheeled mobile robot to verify the effectiveness of the proposed method.

\section{Introduction}

As a branch of robotics research field, the mobile robot increasingly plays a significant role in various areas. Now a number of researchers are studying SLAM (simultaneous localization and mapping) technology. It uses the combination of ordinary camera, and resolves the depth information of environment through the complex arithmetic, then uses the depth information to study SLAM. It is feasible that using binocular vision to analyze the depth information, but compared with sensor that straightforward obtains depth information is more complex, and the hardware requirements of binocular vision are relatively higher, and is vulnerable to be interfered by external environmental factors, which can form noise or distortion error, is not conducive to improve the accuracy of the results. However, Kinect solves the puzzle, because it can directly get the depth information, avoid a lot of unnecessary trouble ${ }^{[1]}$. This paper studies the navigation method of mobile robot based on Kinect depth information, including the SLAM and path planning.

\section{Kinect Depth Imaging}

Kinect can obtain depth information cheaply and efficiently and form the depth image to represent the surrounding environment by collecting all the line of sight range of points. Without considering the color or within the local space, depth image can replace binocular imaging ${ }^{[2]}$.
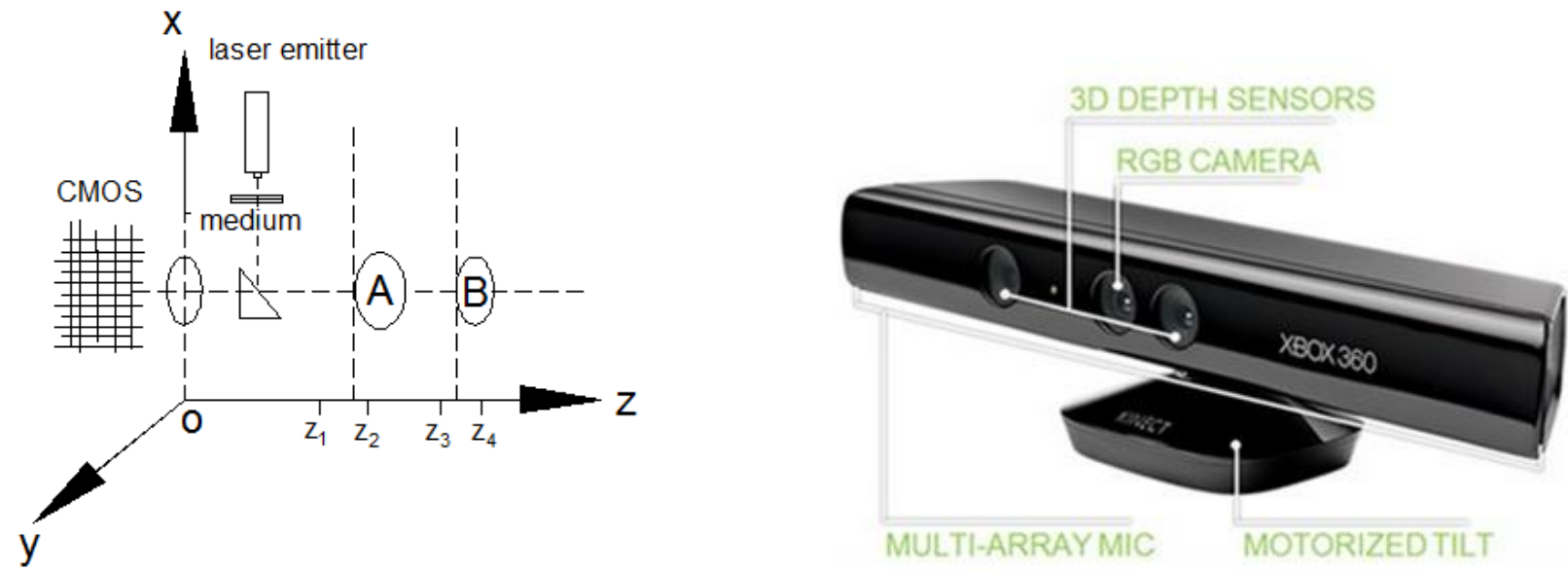

Fig. 1 The Depth Imaging Principle and Kinect

The depth imaging principle of the Kinect is optical encoding technology. Imaging system has three core components: laser emitter, uneven transparent medium, CMOS device. The structure of the imaging system and Kinect are showed in figure 1. Uneven transparent medium is in front of the laser 
emitter. Uneven transparent medium and CMOS device are facing the target scene with a certain angle. The laser projected from the laser emitter passes through the uneven transparent medium and then laser speckle appears. CMOS sensor can shoot speckle image.

Kinect sensor can obtain 2D depth map by scanning, including the distance information and its corresponding pixel coordinates between the objects and Kinect. Quantify the point cloud data for later calculation and measurement. Quantitative process can convert 2D depth map into 3D data (in the world coordinate system) and convert the data from the image coordinate to the world coordinate system.

\section{The Kinematic Model}

As is shown in Figure 2, two centers coincide with the center gravity of the car at $\mathrm{C}$ point. Two axis are perpendicular to the traveling direction of the mobile robot, which are also parallel to the $Y_{R}$ axis in the local coordinate system at a time. In addition, in the same coordinate system, $r$ is the radius of the drive wheel, $L$ is the distance between the two centers, $\theta$ is the angle between $X_{R}$ axis and $X$ axis which in the global coordinate system. The local coordinate system attaches to the robot, while the global coordinate system is stationary.

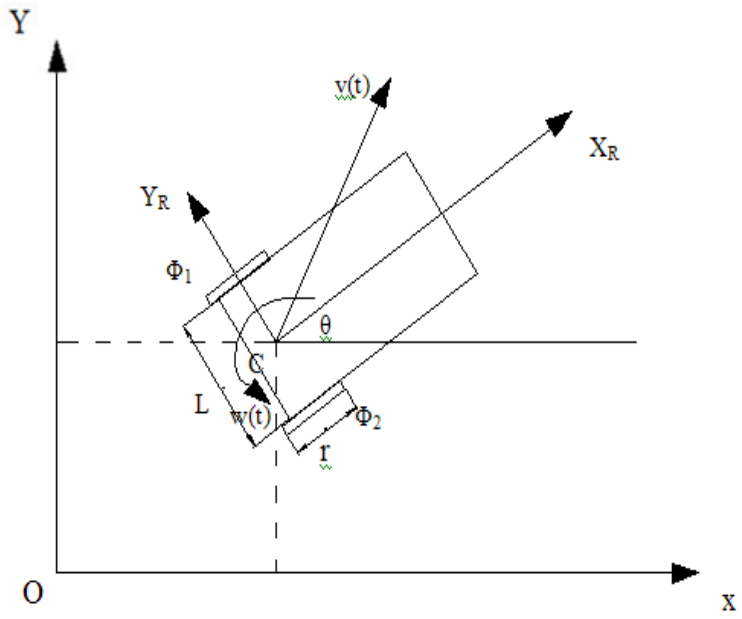

Fig. 2 The Kinematics Model

Ignore some factors (such as differences tire elasticity and surface friction) which have an affect on the moving speed of the wheel and focus on the analysis of speed, turning radius, angle and average speed. In the process of turning, set the angle speed as $\omega$, the speed of the two centers as $\varphi_{1}$ 、 $\varphi_{2}$, the linear velocity of the center of mass as $V_{C}$, the linear velocities of the two centers as $V_{L} 、 V_{R}$,

Ignore the sliding friction between the two centers and the ground, the following formulas can be obtained with the given parameters of $L, \theta, R$ :

$$
\begin{aligned}
& \left\{\begin{array}{l}
\mathrm{V}_{\mathrm{n}}=\left(\mathrm{V}_{\mathrm{R}}+\mathrm{V}_{\mathrm{L}}\right) / 2 \\
\omega=\left(\mathrm{V}_{\mathrm{R}}-\mathrm{V}_{\mathrm{L}}\right) / L
\end{array}\right. \\
& 2 \pi(\mathrm{R}-\mathrm{L} / 2) / V_{l}=2 \pi(\mathrm{R}+\mathrm{L} / 2) / V_{R} \\
& R=\frac{\mathrm{L}}{2}\left(\frac{V_{r}+V_{l}}{V_{r}-V_{l}}\right)
\end{aligned}
$$

Selecting the speed $V_{L}, V_{R}$,of the two centers as the moving condition input of the moving robot, and the center state matrix $\xi_{p}=(X, Y, \theta)^{T}$ as the output, the state of the mobile robot can be solved with the following equation (3-4) at any time: 


$$
\xi_{p}=[X(t), Y(t), \theta(t)]^{T}=\left|\begin{array}{c}
X_{0}+\int_{0}^{t} \frac{V_{R}+V_{L}}{2} \cos \theta d t \\
Y_{0}+\int_{0}^{t} \frac{V_{R}+V_{L}}{2} \sin \theta d t \\
\theta_{0}+\int_{0}^{t} \frac{V_{R}-V_{L}}{D} d t
\end{array}\right|
$$

In order to get the movement state of the mobile robot, the correspondence relationship between the global coordinate system and local one needs to be found, and use the relationship to achieve mutual conversion between the two coordinate systems. If the mobile robot in the global coordinate system values is $\left(x_{c}, y_{c}, \theta_{c}\right)$, and the space coordinate of a point in the local one is $\left(x_{1}, y_{1}\right)$, then the specific coordinates $\left(x_{g}, y_{g}\right)$ of this point in the global coordinate system can be obtained using equation (3-5):

$$
\left\{\begin{array}{l}
x_{\mathrm{g}}=x_{c}+X_{i} \cos \theta_{\mathrm{c}}-y_{l} \sin \theta_{\mathrm{c}} \\
y_{\mathrm{g}}=y_{c}+x_{i} \sin \theta_{\mathrm{c}}-y_{l} \cos \theta_{\mathrm{c}} .
\end{array}\right.
$$

\section{Navigation}

SLAM is the simultaneous localization and mapping. This proposed concept serves the navigation system of mobile robot. The concept takes a big part of the navigation system. Self-localization is to estimate the current position of moblie robot through surrounding environment information collected by certain sensors. Then using the information to bulid electronic maps of environment to realize the autonomous motion. A lot of mobile robot navigation systems need SLAM, so its importance is known to all ${ }^{[3]}$.

\section{Self-localization}

Self-localization of mobile robot is based on movement. The kinematics model set up before is to estimate the real time location to realize localization of mobile robot. The state of motion of differential drive mobile robot is determined by the speed of the two driving wheels, the driving wheel track, and the driving wheel radius. The speed of left drive wheel is $\omega_{1}$, the speed of right drive wheel is $\omega_{2}$, the driving wheel radius is $R$, the distance between two wheels is $L$.

The mobile robot's current sampling period is $\Delta T$ (the sampling period is for the encoder value cycle of mobile robot), in the period $\Delta T$, the distance $\Delta X$ on the direction of $X$ is:

$$
\Delta x=\frac{\Delta s_{1}+\Delta s_{2}}{2} \cos \left(\theta+\frac{\Delta \theta}{2}\right)
$$

$\Delta S 1$ is the incremental motion of left drive wheel, $\Delta s_{1}=R \omega_{1} \Delta T$, and $\Delta S 2$ is the incremental motion of right drive wheel, $\Delta s_{2}=R \omega_{2} \Delta T ; \theta$ is the angle from the original location $p(x, y, \theta)$ of mobile robot. $\Delta \theta=\frac{\Delta s_{2}-\Delta s_{1}}{L}$ is the rotary motion increment of the mobile robot. And the distance $\Delta Y$ on the direction of $Y$ is:

$$
\Delta y=\frac{\Delta s_{1}+\Delta s_{2}}{2} \sin \left(\theta+\frac{\Delta \theta}{2}\right)
$$

The following equation (3-8) is a mobile robot odometer estimation model. The current mobile robot real-time position $p$ ' is equal to the sum of the initial position of the mobile robot $p(x, y, \theta)$ and incremental motion of mobile robot $(\Delta x, \Delta y, \Delta \theta)$.

$$
\mathrm{P}^{\prime}=\left[\begin{array}{l}
x^{\prime} \\
y^{\prime} \\
\theta^{\prime}
\end{array}\right]=\left[\begin{array}{l}
x \\
y \\
\theta
\end{array}\right]+\left[\begin{array}{l}
\Delta x \\
\Delta y \\
\Delta \theta
\end{array}\right]
$$




\section{Map-building}

For the navigation system which is in unknown environment, the first is how to continue to obtain the environmental characteristics information from an unknown point of origin through some sensors (laser, vision, ultrasonic), and gradually establish a map of the surrounding environment. Map representation of the operating environment is an indispensable part in the navigation system, and it is not only the basis for subsequent path planning, but also is a key step which makes the location of the mobile robot get practical sense. Based on the current status of research, the main method to create an electronic map of the environment are three kinds: raster modeling method, visual method and topological modeling method ${ }^{[4]}$.

Most implementations of SLAM technology by vision system are based on feature extraction and feature matching manner. After depth sensor point cloud data is acquired by Kinect, and make some purification and extract corner features of the depth images of different frames, and then matching features, and finally build the map.

\section{Path planning}

Path planning is a kind of process that AGV searches the most rational path after orientation and creating environment map ${ }^{[5]}$. Through this process, AGV could find the shortest path between initial and objective location while rational avoiding barriers. Artificial potential field, Dijkstra algorithm, A* algorithm, deep optimization algorithm, and breadth first algorithm are the most distinguished algorithms. A* algorithm, which are simple, high calculating efficiency, and great real-time performance, are used to realize path planning in this paper.

In the state space corresponding to the node graph, the process of path searching continuously cycles. The system finds the optimal searching location through assessing the nodes in each cycle. This optimal searching location will be starting point of the next cycle. System will find the target point after continuously cycles at last. This approach deletes some unnecessary searching path that makes it high efficiency ${ }^{[6]}$.

\section{Simulation}

Simulation is performed with a mobile robot with Kinect in ROS system, figure 3 is the result of a map created by the robot in the room, as is shown in the grid map, white point is the mobile robot, yellow line is the actual path while green line is the planning path, the outer green thick lines represent the scanned obstacles.

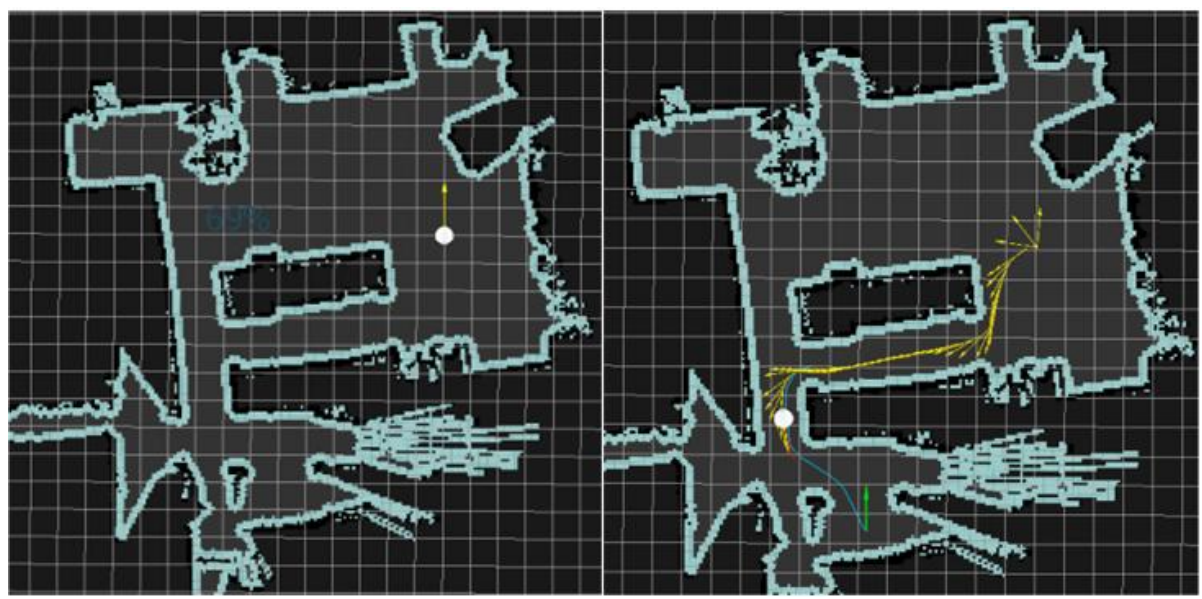

Fig.3 Map Building Experiment

Path planning is applied on the mobile robot and the simulation results are shown in figure 4. Searching process with node graphs is simulated first, and then path is planned though constructing environment map. The left node simulation diagram represents the searching order of nodes using $\mathrm{A}^{*}$ algorithm, while the right diagram represents the path searched out in the planning simulation.

The simulation results prove the effectiveness of the proposed navigation method. 

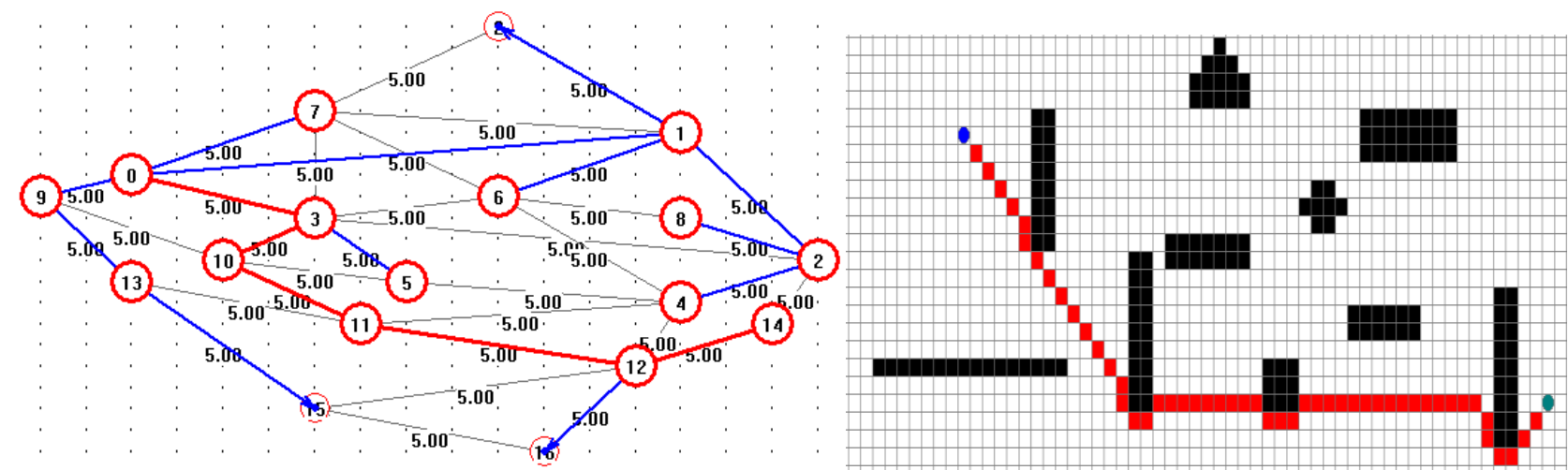

Fig.4 A* Path Planning Simulation

\section{Conclusion}

Depth camera Kinect produced by Microsoft is used as a sensor in this paper. Navigation method used in differential driving mobile robot is investigated. In this paper, the 3D depth information is captured by Kinect; the self-orientation of mobile robot is realized by range estimation model; and two-dimensional electronic map of indoor environment is established. $\mathrm{A}^{*}$ algorithm is used to realize the path planning of mobile robot.

\section{Acknowledgements}

This work was financially supported by the National Natural Science Foundation of China (No. 51405236), part from the Fundamental Research Funds for the Central Universities (No. 22A201514025) and the Open Project Program of Key Laboratory of Intelligent Perception and Systems for High-Dimensional Information of Ministry of Education (No.JYB201506), Shanghai Education Development Foundation Alliance Program (No. LM201404).

\section{References}

[1]Cristóbal Bellés,Filiberto Pla*. A Kinect - Based System for 3D Reconstruction of Sewer

Manholes[J]. Computer - Aided Civil and Infrastructure Engineering, 2015,30(11), 906-917.

[2]Khazaeli, Mehdi,Javadpour, Leili,Knapp, Gerald M. Kinect Applications in Construction: from Tracking to Reconstruction[J]. IIE Annual Conference. Proceedings,2013, 259-267 .

[3]Chunguang Li,Chongben Tao,Guodong Liu,Erik Cuevas. 3D Visual SLAM Based on Multiple Iterative Closest Point[J]. Mathematical Problems in Engineering,2015, Vol.2015,1-11.

[4]Khoshelham Kourosh,Elberink Sander Oude. Accuracy and resolution of Kinect depth data for indoor mapping applications.[J]. Sensors,2012,12(2) 1437-1454.

[5]Li-xia Zhang,Shao-ting Zhang,Hong-zhi Xie,Xia-hai Zhuang,Li-xu Gu. Kinect-based automatic spatial registration framework for neurosurgical navigation[J]. Journal of Shanghai Jiaotong University (Science),2014,19(5), 617-623.

[6]Yang Gao,Shu-dong Sun,Da-wei Hu,Lai-jun Wang. An online path planning approach of mobile robot based on particle filter[J]. Industrial Robot: An International Journal,2013,40(4). 